\title{
Structured Data Acquisition in Oncology
}

\author{
Maurice Henkel Bram Stieltjes \\ Department of Radiology, University Hospital Basel, Basel, Switzerland
}

\section{Keywords}

Data element $\cdot$ Electronic health record $\cdot$ Structured reporting $\cdot$ Support decision-making $\cdot$ Data stream

\begin{abstract}
Background: Demographic changes and improvement in therapy have shifted the focus of treatment towards chronic diseases and multiple health conditions. This has caused a tremendous increase in data per patient that needs to be integrated longitudinally and across departmental silos. The general increase in the volume of data per diagnostic examination and the number of diagnostic procedures per diagnostic pathway additionally accentuate this data integration challenge. Summary: Subspecialization in medicine has led to largely autonomously organized departments with independent IT ecosystems. This patchwork of IT infrastructure is not prepared to meet the data integration challenge. The resulting lack of integrated information makes the treatment of chronically ill patients increasingly difficult and error prone. Key Message: A sustainable method for data acquisition is needed to aid multimodal treatment and improve efficiency in healthcare.

๑) 2019 S. Karger AG, Basel
\end{abstract}

\section{Introduction}

To understand the challenges of the hospitals' infrastructure today, it is helpful to look at its development. During the 19th century, physicians began to specialize and professionalize. This was necessary to protect them- selves against unqualified competitors [1]. A central element of professionalization is a high degree of autonomy and self-determination. The practice of medicine changed in the face of the technology-driven improvements in surgery and patient care [2]. Physicians began to organize themselves according to organs, patient groups, therapeutic techniques, and national needs. Since their beginning, these origins of modern professions have struggled to define their identity, values, sphere of activity, and role in patient care. To ensure that its members had shared experiences, values, solutions, and the language for professional tools, the disciplines increasingly worked in si$\operatorname{los}[3,4]$.

This has led to different levels of development in terms of digitalization between the disciplines and between hospitals. Advances in science created a constant demand for restructuring of processes and new requirements for the department's information technology (IT). As a result, the IT infrastructure evolved with almost the same degree of specialization as the departments. The resulting patchwork is now a hospital's administrative, cultural, and technical infrastructure. Despite the advantages of structured data entry, narrative text remained the preferred way of data acquisition in healthcare. This way of data acquisition and the customization of existing information systems makes the access and usability of medical data tremendously complicated [5-8].

\section{Rising Patient Complexity}

The changing spectrum of diseases increasingly poses new challenges for the management of medical data. Improved healthcare, socioeconomic conditions, and living

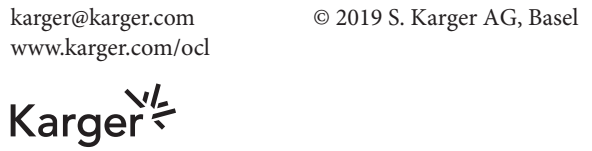

Maurice Henkel

Department of Radiology, University Hospital Basel Spitalstrasse 2

$\mathrm{CH}-4031$ Basel (Switzerland)

E-Mail mauricejohannes.henkel@usb.ch 
standards have led to an ageing society [9]. Improved treatment allows patients to survive with their diseases for prolonged periods of time and to acquire multiple conditions $[10,11]$. These multiple chronic conditions require continuous interdisciplinary management like never before in medical history $[10,12]$. Specialized departments provide precise diagnostics with large amounts of data for the patient's multiple conditions. Since this information is obtained in largely encapsulated IT ecosystems, a comprehensive view of the patient's increasingly complex condition becomes a rising problem [5, 13]. Missing standards in data acquisition and management prevent the efficient use of data and lead to most decisions being based on expert consensus $[14,15]$. The lack of coordination of patient care, information deficits, overtreatment, and the increasing complexity of administration have led to an enormous increase in health expenditure and will continue unless we improve information management $[16,17]$.

Good data management is a prerequisite for knowledge discovery and subsequent data and knowledge integration to improve patient care. The difficulty to gain the required high-quality medical knowledge for evidencebased treatment depends on the ability of high-quality data [18]. Rational data acquisition and management is a key challenge to ensure the quality of the treatment, a streamlined workflow, and a sustainable way of financing the healthcare system [19]. Integrative solutions for data acquisition are needed to adapt information to these different requirements and purposes. Providers of health information technology are confronted with completely new challenges. Instead of protecting their products, open systems and standards are required to support information exchange.

\section{The Hospitals' IT Patchwork}

After decades of professionalization and specialization, departments customized their processes according to their needs. Cultural, administrative, and technological barriers have emerged that have induced complex and inefficient workflows. Clinical oncology is a dynamic, multidisciplinary healthcare specialty with complex decision-making and care coordination needs [20] and, as such, a good example for a discipline that faces the aforementioned challenges. This field clearly reveals the implications of the hospitals' insufficient infrastructure.

To understand these complicated processes, we analyzed our diagnostic workflow with an external consult- ing group (Fig. 1). The aim of this analysis was to overcome the functional silos, strengthen teamwork, and improve diagnostic pathway. Using the diagnostic workup in prostate cancer as an example, we analyzed the processes of our diagnostic departments from the first request in radiology to the pathologically confirmed diagnosis. The individual steps in this diagnostic process were examined for gaps, actors involved, standard procedures, deliverables, resources, and duration. Involved in the analysis were two radiologists, two pathologists, one urologist, the $\mathrm{VC}$ of research, a technical assistant, as well as two consultants. Starting from the radiological request, we identified 16 individual triggered steps in the entire workflow to the final diagnosis. These numerous steps have evolved through changing demands and require manual intervention. A lack of standardization, e.g. for image requests, requires manual information transfer to the radiological information system. Missing information in this request prevents efficient scheduling of radiological examinations and may require manual requests of further information from the referring physician. The necessity of these manual interventions affects the entire diagnostic pathway and can cause various errors. In our analysis we identified 28 independent gaps. Most of these gaps concern our information management. The use of multiple, siloed information systems which poorly exchange information causes an information deficit at various points. The resulting lack of integrated information leads to fragmented patient data and prevents a comprehensive view of the patient's general condition. As a result, seven actors with numerous, uncoordinated, and undocumented contacts are involved in the diagnostic process to obtain a comprehensive view on patient's data for a valid diagnosis. Since the result of this manual effort is not documented digitally, it needs to be repeated for every single examination.

This situation is aggravated through an environment of constantly changing teams and high time pressure, common to a teaching hospital setting. Repeated examinations and postponements of patients to the next multidisciplinary teams (MDT) conference are the result.

Through this analysis we were able to understand the key problems in our workflow. The expanding scale and complexity of healthcare challenges efficient workflow and communication. Department-internal organization of processes and the patchwork IT infrastructure prevent a linear workflow in which steps are systematically initiated. Instead, the patient's information is fragmented into several isolated IT ecosystems that disrupt information exchange and comprehensive evaluation. This is a crucial 


\section{Analysis of the Initial State}

Process Mapping

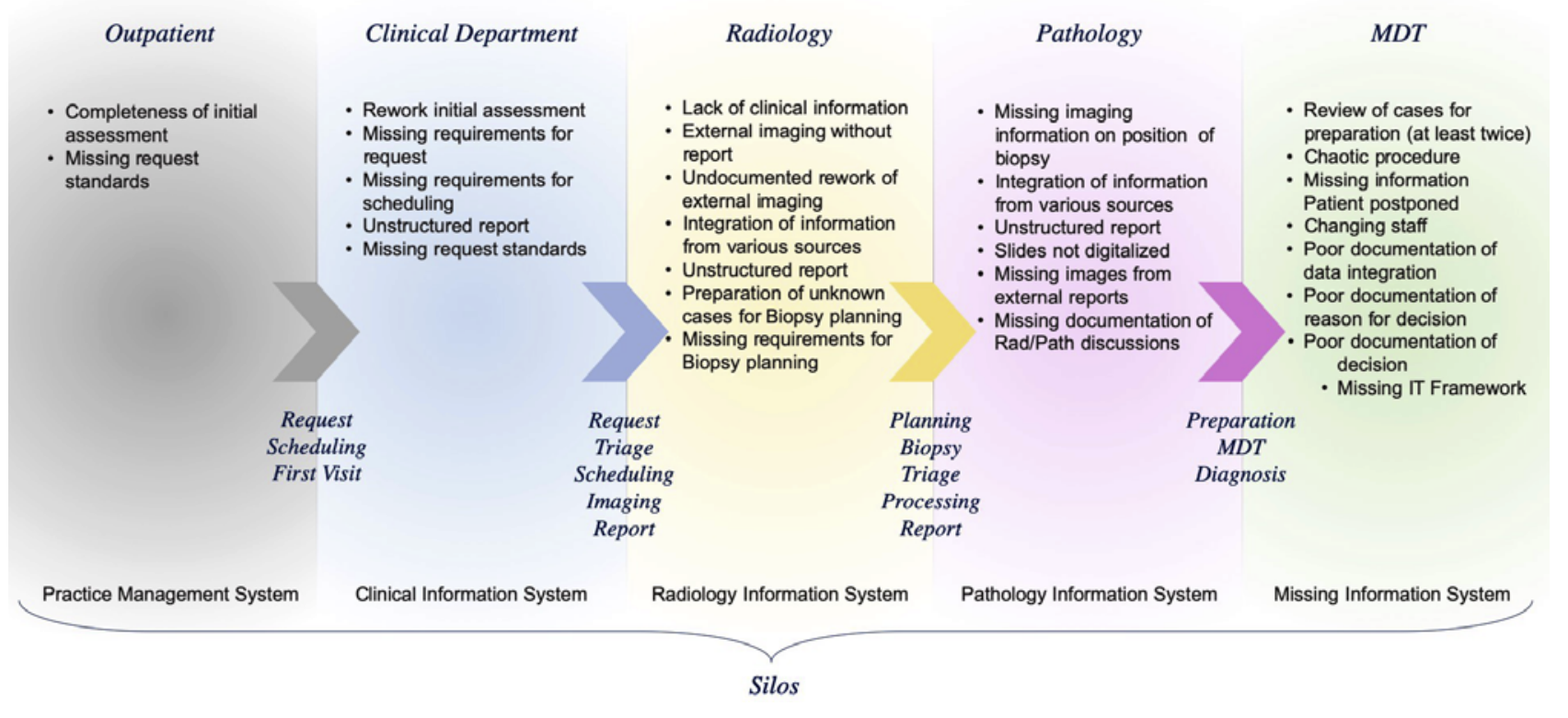

Fig. 1. Workflow analysis. The workflow analysis shows the diagnostic pathway of a patient to his final diagnosis. Color-coded, the respective, largely independent departments. Each department uses its own information system with its own individual standards for data acquisition and storage. Patient information is fragmented

limitation as the evaluation of medical information highly depends on its context [21]. It is a tremendous effort to keep track of the patient's status, the reason for each investigation and the patient's medical history. Repetitive and uncoordinated interactions between the stakeholders, that disrupt department-specific workflows, are the consequence.

\section{Restriction of Current Solutions}

To ensure sufficient management for complex patient cases despite these obstacles, MDT have been initiated, more reactionary than evolutionary $[10,22,23]$. In MDT conferences, highly specialized professionals meet to provide their expertise for the best possible care. During these conferences, all available information will be merged to get a comprehensive view of the patient's status and to discuss treatment options. However, MDT conferences do not address the underlying problem of inadequate IT infrastructure. The workflow remains inefficient with all the aforementioned limitations and does not ensure completeness of information. The evaluation of patient infor- among these information systems. Due to their difficult accessibility and the lack of uniform data standards, these systems are named as silos. The resulting gaps in the workflow in the respective departments are shown as key points. The arrows indicate the necessary steps during the diagnostic process.

mation continues to require repeated effort for all involved. Since the conference usually takes place at a fixed time during the week, it highly depends on the completeness of the information and the availability of specialists at a certain time point with little or no flexibility. The hospitals' shift system cannot ensure this requirement, which means that important decisions may be postponed to the next conference [24-26].

Vendors in health technology have recognized the rising need for support of MDT conferences [27]. Clinical decision support (CDS) systems are designed to directly support clinical decision-makers by using multi-source information to provide patient-centered assessments or guidance $[28,29]$. There is growing evidence that oncological and nononcological CDS systems appear to be well accepted and may support the diagnostic process, risk assessment, care plan, and treatment [30-35]. However, the implementation of CDS systems entails enormous technical, organizational, and cultural challenges [15]. The patchwork of IT systems customized to different needs in each hospital prevents the accessibility and usability of 


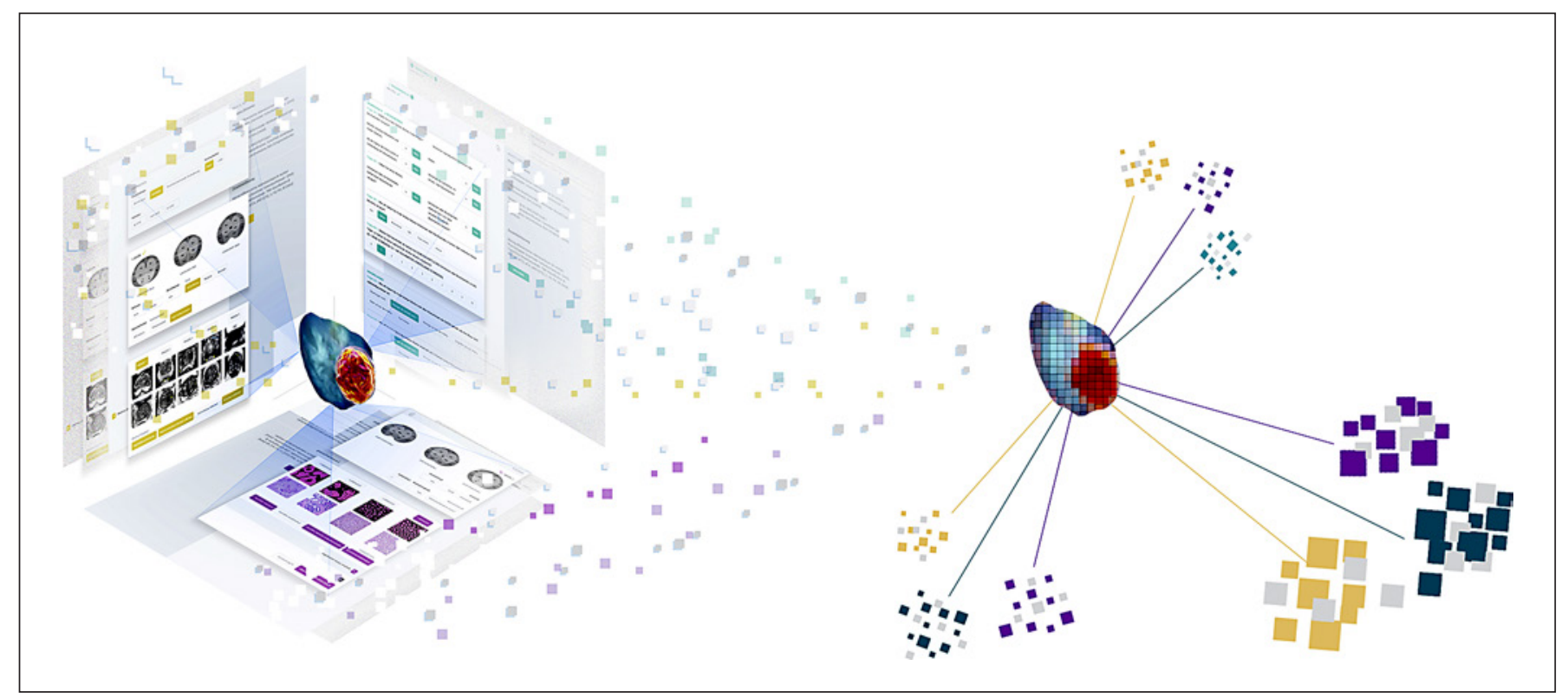

Fig. 2. Prostate cancer workup using data elements. The left picture shows the prostate to be examined in blue with a tumor in red. In the course of the screening, examinations are carried out to determine the tumor identity, stage, and location. All information from these examinations is entered using our forms (forms surrounding the prostate; blue, urology; yellow, radiology; red, pathology). The data elements collected during this diagnostic pathway are merged in our database to form a virtual image of the examined prostate (pixelated prostate on the right). The data elements of this virtual image of the prostate can be regrouped according to the purpose or the recipient (data clouds on the right). the data required for the CDS system. Pure digitization has led to an enormous volume of data, but there is a substantial gap between information volume- and information value increase. Even if the data is accessible, it is necessary to distinguish between digital data and actionable information. The evaluation of medical data is highly complex and depends on various factors. The interpretation of simplest parameters such as body temperature has different meanings, depending on where, when, and how it is measured [36]. Electronic health records (EHR) are currently the most compressed extracts from these complex and heterogeneous medical data. EHRs are used for data exchange, but even within the same hospital they are fragmented at best. There are no common standards for the structuring of EHR and the way it is stored is not compatible with most applications and technologies [37]. On the contrary, many EHRs are little more than organized PDF repositories lacking a strong, data element-oriented content. On this basis, the implementation opportunities and scalability of CDS systems are challenging.

The most important challenge in obtaining actionable information from existing data is how to structure it. Deep learning methods such as natural language processing (NLP) are used to gain valuable information from electronic health records [38]. This computational approach tries to extract data elements from the report and to transfer them into a machine-readable format [6]. Despite successful application in this field, this approach faces several limitations [39]. The gain in valuable information depends on the quality of the underlying reports [18, 40]. Since every examination is independently evaluated, a high intellectual effort is required to provide the intended context. The customization of the EHR system in each department causes the data in one hospital to look different than the data of a similar patient receiving similar treatment in another hospital [5-8]. The resulting differences in relation, meaning, and purpose make it tremendously difficult to evaluate the data in its intended context and gain valuable information from it $[41,42]$.

\section{The Need for Sustainable Data Management}

A more sustainable method of data acquisition is a central requirement to meet the rapid changes in healthcare needs. This sustainable method primarily requires a cultural change. Departments must no longer define their processes and standards as independent as before. Standards need to be defined on the highest level to enable cooperation within and across hospitals. The most im- 
Fig. 3. Data elements providing multiple outputs. By visualizing the most important risk factors and the Quality of Life Index, lucid charts can be created to support the decision-making process (top). Synoptic reporting is used to combine data elements with text modules to create an explanatory text. This method allows us to use text modules that are adapted to the recipient. In this way a traditional report for the specialist, a summarizing report for the general practitioner, and a comprehensible report for the patient can be created at the same time.

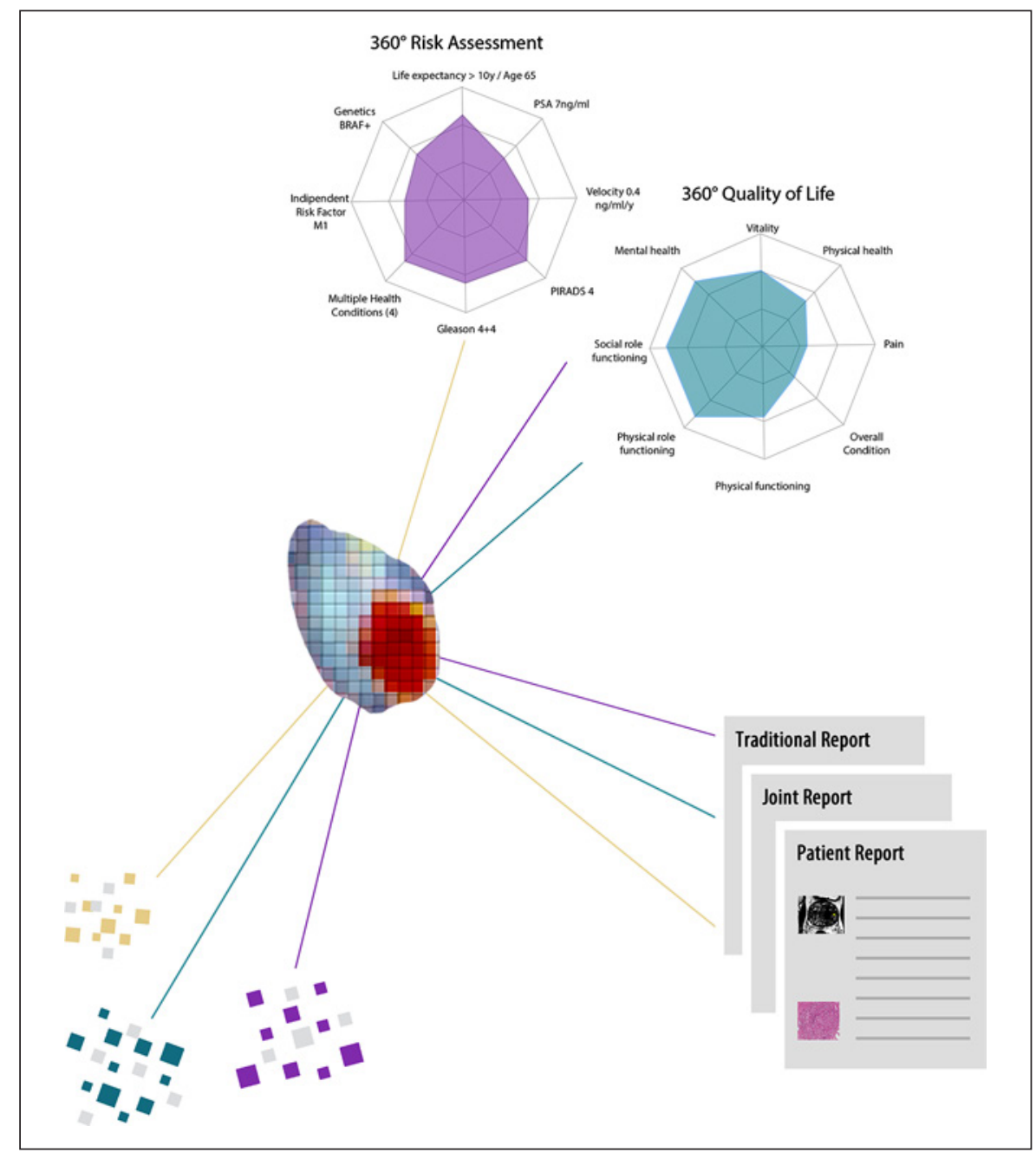

portant part of these standardizations concerns the way of data acquisition. These standards need to ensure the acquisition of high-quality and functionally usable data to streamline processes, improve teamwork, and provide valuable information for the best possible treatment decisions. Given the complexity of health data and the enormous amount of patient information, the definition of data elements and their relation should be implemented at the stage of its acquisition. This is a central paradigm to ensure that data is available in various formats for multiple purposes and usable in a variety of information systems [19].

A promising approach is the use of synoptic reporting [43]. Instead of writing highly variable, narrative reports, a standardized form is filled out and the collected data elements are used to create an entire report. This way of documentation is suitable for different purposes like medical history, radiological and histological examina- tions and has already shown that the reports are more accurate, clearer, and more coherent than narrative reports [44]. The usage of this data element-oriented way of data acquisition ensures for the first time that patients' data is not only available, but usable. If this approach is applied to all examinations necessary in the context of a specific disease, information could be integrated during the diagnostic pathway instead of being fragmented into multiple information systems. Thus, an integrated view from multiple perspectives would be possible (Fig. 2). To obtain valuable information from data elements, they need to be linked along the patient's pathway and comprehensively integrate the full diagnostic and therapeutic process. However, the segmentation of findings and diagnoses into their features varies from previously known data such as body temperature or blood pressure.

Using data elements for data acquisition, the description of a certain finding, for instance in an MRI of the 
prostate, is divided into its describing features. A suspicious lesion would be divided into several elements like localization, diameter, shape, relation to the surrounding anatomic structures, signal behavior, etc. Related parameters, such as the Gleason score, that are collected along the diagnostic pathway need to be assigned to this certain finding. That would allow to combine all available elements and to create a digital twin of the examined object (e.g., prostate). To avoid erroneous mapping of data elements, a database design is required that is able to group data elements according to their relationship in the intended context.

The use of data elements and predefined relationships ensure the reuse of information for various purposes. The information can be reassembled in different variants according to the requirements of the respective recipient. The use of synoptic reports will allow the use of traditional reports for information exchange between highly specialized professionals. Detailed, but conclusively summarized joint reports can be created for general practitioners. Instead of text modules that are appropriate for health professional, text modules that correspond to the patient's level of knowledge could be used for a patient report. Tumor boards require a well-structured preparation of relevant information for an efficient decisionmaking process. Data elements can be used to visualize relevant information such as risk factors and the general condition of the patient in lucid diagrams for targeted support (Fig. 3).

The implementation of standardized data acquisition based on data elements pave the way for a uniform data flow to streamline processes across departments. Predefined thresholds in an examination could trigger automatically subsequent steps (e.g., additional examinations, discussions between actors). Thresholds in following examinations would trigger further investigations until the information for the treatment decision is complete. Instead of initiating each step manually, the generated data elements would trigger a process that will continue until the final diagnosis is made and guide the patient through his or her hospital journey. The patient's data stream would help to understand why which step was initiated, what the patient's current status is and what the next steps would be. The availability of data elements instead of rigid reports allows to continuously evaluate each examination and the complete diagnostic pathway. If patients are divided into the wrong risk groups or if an alternative pathway shows better performance, the processes could be adapted accordingly. Thus, decisions and processes could be made on a data-driven basis.

\section{Conclusion}

Existing structures in the hospital are facing new challenges caused by demographic transition and increasing complexity of data acquisition and treatment decisions. The strict division into departments with their respective IT ecosystems is not a suitable strategy to provide efficient interdisciplinary management. It requires a cultural change in which previously autarkic governed departments adhere to general standards in order to achieve a higher goal. The way in which data is collected needs to change fundamentally in order to adapt to changing healthcare needs. Data can no longer be acquired in the form of a rigid, dedicated report, which prohibits the reusability for various needs and purposes. An appropriate infrastructure is required to ensure efficient data management using data elements. These are fundamental requirements for a sustainable healthcare system that is able to adapt to the rapid changing demands of society and the environment.

\section{Acknowledgement}

We thank Jonas Laux, MSc, and Matthias Klan, MSc, for technical guidance, and Ramon Höcker for the graphical input that greatly improved the review.

\section{Disclosure Statement}

The authors have no conflicts of interest to declare.

\section{Funding Sources}

Novartis Freenovation Award to B.S.
1 Waddington I. The movement toward the professionalization of medicine. BMJ. 1990; 301:688-90.

2 Lerman C, Jameson JL. Leadership Development in Medicine. N Engl J Med. 2018 May; 378(20):1862-3.

3 Hall P. Interprofessional teamwork: Professional cultures as barriers. J Interprof Care. 2005 May; 19 Suppl 1:188-96.

4 Cruess SR, Johnston S, Cruess RL. Professionalism for medicine: opportunities and obligations. Med J Aust. 2002 Aug;177(4):208-11.

5 Svensson A, Larsson L, Hansson A, editors. IT Systems for Collaboration in Healthcare for Frail Elderly People. European Conference on Knowledge Management. Academic Conferences International Limited; 2016. 
6 Rajkomar A, Oren E, Chen K, Dai AM, Hajaj $\mathrm{N}$, Hardt M, et al. Scalable and accurate deep learning with electronic health records. npj Digital Medicine. 2018;1(1):18.

7 Adam-Ledunois S, Damart S, Lacroux A. The healthcare organization seen as composed of silos: a relevant representation? Post-Print hal-01891447, HAL. 2018.

8 Halvorsrud R, Lillegaard AL, Røhne M, Jensen AM. Managing Complex Patient Journeys in Healthcare. In: Pfannstiel MA, Rasche C, editors. Service Design and Service Thinking in Healthcare and Hospital Management: Theory, Concepts, Practice. Cham: Springer International Publishing; 2019. pp. 329-46.

9 Zaidi B, Morgan SP. The second demographic transition theory: A review and appraisal. Annu Rev Sociol. 2017 Jul;43(1):473-92.

10 Barnett K, Mercer SW, Norbury M, Watt G, Wyke S, Guthrie B. Epidemiology of multimorbidity and implications for health care, research, and medical education: a cross-sectional study. Lancet. 2012 Jul;380(9836):3743.

11 Wallace E, Salisbury C, Guthrie B, Lewis C, Fahey T, Smith SM. Managing patients with multimorbidity in primary care. BMJ. 2015 Jan;350:h176.

12 Marengoni A, Angleman S, Melis R, Mangialasche F, Karp A, Garmen A, et al. Aging with multimorbidity: a systematic review of the literature. Ageing Res Rev. 2011 Sep;10(4):4309.

13 Tang V, Siu PK, Choy KL, Lam HY, Ho GT, Lee $\mathrm{CK}$, et al. An adaptive clinical decision support system for serving the elderly with chronic diseases in healthcare industry. Expert Syst. 2019;36(2):e12369.

14 Califf RM, Robb MA, Bindman AB, Briggs JP, Collins FS, Conway PH, et al. Transforming Evidence Generation to Support Health and Health Care Decisions. N Engl J Med. 2016 Dec;375(24):2395-400.

15 Tricoci P, Allen JM, Kramer JM, Califf RM, Smith SC Jr. Scientific evidence underlying the ACC/AHA clinical practice guidelines. JAMA. 2009 Feb;301(8):831-41.

16 Thorpe KE, Allen L, Joski P. The Role of Chronic Disease, Obesity, and Improved Treatment and Detection in Accounting for the Rise in Healthcare Spending Between 1987 and 2011. Appl Health Econ Health Policy. 2015 Aug;13(4):381-7.

17 De Pietro C, Camenzind P, Sturny I, Crivelli L, Edwards-Garavoglia S, Spranger A, et al. Switzerland: Health System Review [xix.]. Health Syst Transit. 2015;17(4):1-288.

18 Cai T, Giannopoulos AA, Yu S, Kelil T, Ripley B, Kumamaru KK, et al. Natural Language Processing Technologies in Radiology Research and Clinical Applications. Radiographics. 2016 Jan-Feb;36(1):176-91.
19 Wang Y, Kung L, Wang WY, Cegielski CG. An integrated big data analytics-enabled transformation model: application to health care. Inf Manage. 2018;55(1):64-79.

20 Clauser SB, Wagner EH, Aiello Bowles EJ, Tuzzio L, Greene SM. Improving modern cancer care through information technology. Am J Prev Med. 2011 May;40(5 Suppl 2): S198-207.

21 Eysenbach G, Diepgen TL. Towards quality management of medical information on the internet: evaluation, labelling, and filtering of information. BMJ. 1998 Nov; 317(7171): 1496-500.

22 Pefoyo AJ, Bronskill SE, Gruneir A, Calzavara A, Thavorn K, Petrosyan Y, et al. The increasing burden and complexity of multimorbidity. BMC Public Health. 2015 Apr;15(1):415.

23 Taylor C, Munro AJ, Glynne-Jones R, Griffith C, Trevatt P, Richards M, et al. Multidisciplinary team working in cancer: what is the evidence? BMJ. 2010 Mar;340:c951.

24 Basta YL, Baur OL, van Dieren S, Klinkenbijl $\mathrm{JH}$, Fockens P, Tytgat KM. Is there a Benefit of Multidisciplinary Cancer Team Meetings for Patients with Gastrointestinal Malignancies? Ann Surg Oncol. 2016 Aug;23(8):24307.

25 Lanceley A, Savage J, Menon U, Jacobs I. Influences on multidisciplinary team decisionmaking. Int J Gynecol Cancer. 2008 Mar-Apr; 18(2):215-22.

26 Horstead A, Cree A. Achieving transparency in forensic risk assessment: a multimodal approach. Adv Psychiatr Treat. 2013;19(5):3517.

27 Prades J, Remue E, van Hoof E, Borras JM. Is it worth reorganising cancer services on the basis of multidisciplinary teams (MDTs)? A systematic review of the objectives and organisation of MDTs and their impact on patient outcomes. Health Policy. 2015 Apr;119(4): 464-74.

28 Lobach D, Sanders GD, Bright TJ, Wong A, Dhurjati R, Bristow E, et al. Enabling health care decisionmaking through clinical decision support and knowledge management. Evid Rep Technol Assess (Full Rep). 2012 Apr;203(203):1-784

29 Castaneda C, Nalley K, Mannion C, Bhattacharyya P, Blake P, Pecora A, et al. Clinical decision support systems for improving diagnostic accuracy and achieving precision medicine. J Clin Bioinforma. 2015 Mar;5(1):4.

30 Ruland CM, White T, Stevens M, Fanciullo G, Khilani SM. Effects of a computerized system to support shared decision making in symptom management of cancer patients: preliminary results. J Am Med Inform Assoc. 2003 Nov-Dec;10(6):573-9.

31 Blum D, Raj SX, Oberholzer R, Riphagen II, Strasser F, Kaasa S, et al. Computer-Based Clinical Decision Support Systems and $\mathrm{Pa}$ tient-Reported Outcomes: A Systematic Review. Patient. 2015 Oct;8(5):397-409.
32 Damiani G, Pinnarelli L, Colosimo SC, Almiento R, Sicuro L, Galasso R, et al. The effectiveness of computerized clinical guidelines in the process of care: a systematic review. BMC Health Serv Res. 2010 Jan;10(1):2.

33 Tarver WL, Menachemi N. The impact of health information technology on cancer care across the continuum: a systematic review and meta-analysis. J Am Med Inform Assoc. 2016 Mar;23(2):420-7.

34 Myers RE, Stello B, Daskalakis C, Sifri R, González ET, DiCarlo M, et al. Decision Support and Navigation to Increase Colorectal Cancer Screening Among Hispanic Patients. Cancer Epidemiol Biomarkers Prev. 2019 Feb;28(2):384-91.

35 Pawloski PA, Brooks GA, Nielsen ME, OlsonBullis BA. A Systematic Review of Clinical Decision Support Systems for Clinical Oncology Practice. J Natl Compr Canc Netw. 2019 Apr;17(4):331-8.

36 Ismail A, Shehab A, El-Henawy IM. Healthcare Analysis in Smart Big Data Analytics: Reviews, Challenges and Recommendations. In: Hassanien AE, Elhoseny M, Ahmed SH, Singh AK, editors. Security in Smart Cities: Models, Applications, and Challenges. Cham: Springer International Publishing; 2019. pp. 27-45.

37 Kruse CS, Goswamy R, Raval Y, Marawi S Challenges and Opportunities of Big Data in Health Care: A Systematic Review. JMIR Med Inform. 2016 Nov;4(4):e38.

38 Tarpey RJ, Mullarkey MT, editors. Extending Design Science Research Through Systems Theory: A Hospital System of Systems. Extending the Boundaries of Design Science Theory and Practice. Cham: Springer International Publishing; 2019.

39 Zeng Z, Deng Y, Li X, Naumann T, Luo Y. Natural Language Processing for EHR-Based Computational Phenotyping. IEEE/ACM Trans Comput Biol Bioinformatics. 2019 JanFeb;16(1):139-53.

40 Pons E, Braun LM, Hunink MG, Kors JA Natural Language Processing in Radiology: A Systematic Review. Radiology. 2016 May; 279(2):329-43.

41 Frieden TR. Evidence for Health Decision Making - Beyond Randomized, Controlled Trials. N Engl J Med. 2017 Aug;377(5):46575.

42 Weisfeld N, English RA, Claiborne AB. Envisioning a transformed clinical trials enterprise in the United States. Establishing an agenda for 2020: workshop summary. National Academies Press; 2012.

43 Baskovich BW, Allan RW. Web-based synoptic reporting for cancer checklists. J Pathol Inform. 2011 Mar;2:16.

44 Słodkowska J, Cierniak S, Patera J, Kopik J, Baranowski W, Markiewicz T, et al. Functional Assessment of Synoptic Pathology Reporting for Ovarian Cancer. Pathobiology. 2016; 83(2-3):70-8 